\title{
UN ARGUMENTO DAVIDSONIANO CONTRA EL MONISMO ANÓMALO
}

\author{
David Pineda Oliva \\ Departament de Filologia i Filosofia \\ Universitat de Girona \\ david.pineda@udg.es
}

SUMMARY: In this work I offer an argument which shows that Davidson's argument or the claim that mental token events are physical events (Physical Monism) is incompatible with his claim that there are no strict psychophysical or psychological laws (Anomalism of the Mental) and therefore that his wellknown position, Anomalous Monism, is untenable. In the final part of the paper, I draw some important lessons from the inconsistence highlighted which I think help to clarify substantially the options available in the metaphysics of mind.

KEY WORDS: action theory, anomalous monism, mind-body problem, psychophysical laws

RESUMEN: En este trabajo ofrezco un argumento que muestra que el argumento de Davidson a favor de la tesis del monismo físico (que los ejemplares de estados mentales son estados físicos) es de hecho incompatible con la tesis del anomalismo de lo mental (que no hay leyes psicofísicas o psicológicas), y con ello, que la posición conocida como monismo anómalo es en realidad insostenible. En la parte final del artículo extraigo algunas conclusiones importantes de mi argumento, consecuencias que, según creo, ayudan en mucho a clarificar las opciones abiertas en metafísica de la mente.

PALABRAS CLAVE: teoría de la acción, monismo anómalo, problema mente-cuerpo, leyes psicofísicas

En la introducción a la recopilación Essays on Actions and Events, Donald Davidson afirma que la idea fundamental que defenderá en ellos es la tesis de que la noción común de causa que aparece en las teorías científicas y del sentido común acerca de los fenómenos no psicológicos desempeña también un papel esencial en nuestra comprensión de aquello que sea actuar por una razón. ${ }^{1}$

1 Essays on Actions and Events, p. xi. 
En lo que sigue, llamaremos a esta tesis fundamental la tesis T. La tesis T es, a mi juicio, una aportación importantísima a la filosofía de la acción, ampliamente dominada a comienzos de los sesenta por la idea contraria de origen decimonónico según la cual las explicaciones de las acciones intencionadas, por un lado, y las explicaciones de los fenómenos naturales, por otro, serían dos géneros de explicaciones completamente distintos, sin nada sustantivo en común. Mientras las primeras se considerarían eminentemente explicaciones causales, las segundas, esto es, las explicaciones de las acciones, no podrían tener tal consideración al no ser causal el vínculo entre razones y acciones que tales explicaciones exhiben. Davidson rompió en buena medida esta tendencia a ver las cosas de este modo defendiendo la tesis $\mathrm{T}$ con ayuda de un argumento muy convincente presentado y discutido en su famoso trabajo "Actions, Reasons and Causes". Unos años después, en "Mental Events", utilizó la tesis T como una de las premisas para argumentar en favor del monismo anómalo, su conocida teoría filosófica de la mente. Tal teoría tendría como principal objetivo filosófico, según declara el propio autor, ofrecer una respuesta aceptable al viejo y candente problema kantiano de reconciliar la libertad humana con la existencia de leyes naturales que parecen regir el mundo del cual forman parte el hombre y sus actos supuestamente libres y en el cual presumiblemente éste ejerce su libertad.

La solución de Davidson al problema kantiano no pareció muy satisfactoria, y resulta relativamente sencillo hacer ver por qué no lo es una vez presentada; pero el objeto del presente trabajo no es insistir en esta cuestión ya muchas veces debatida, sino intentar mostrar que el argumento davidsoniano que sustenta la tesis T, el argumento de "Actions, Reasons and Causes", no parece compatible con algunos de los argumentos de "Mental Events" que sustentan el monismo anómalo. Dado que la tesis T es una de las premisas que Davidson utiliza para argumentar su posición, lo que pretendo mostrar al fin y a la postre es que el monismo anómalo resulta ser una teoría conceptualmente inestable que oscila entre el dualismo anómalo (la tesis según la cual los acaecimientos mentales no son acaecimientos físicos y no obedecen leyes) y el monismo nomológico (la tesis según la cual 
los acaecimientos mentales son acaecimientos físicos y obedecen leyes físicas o, en su defecto, leyes que dependen de ellas), pero que en cualquier caso no hay ninguna teoría inteligible sustentada por todos los argumentos que presenta Davidson.

Al final del trabajo obtendremos algunas conclusiones filosóficas importantes de nuestra investigación. Se subrayará la importancia del argumento en favor de la tesis $\mathrm{T}$, que tiene mucha más fuerza de la que Davidson pensó que tenía, y se mostrará que la consideración del mismo permite clarificar mucho el panorama de la filosofía de la mente de nuestros días, tanto que el monismo anómalo no tendrá cabida en él.

\section{Las tesis del monismo anómalo}

Para mostrar la inconsistencia que he señalado será necesario analizar con cierto detalle los argumentos que Davidson ofrece para defender su posición. En primer lugar conviene que clarifiquemos debidamente las tesis que dichos argumentos supuestamente tienen que sustentar. Pues bien, el monismo anómalo puede caracterizarse como la conjunción de dos tesis:

(MF) Monismo físico: los acaecimientos mentales son acaecimientos físicos.

(AM) Anomalismo mental: no existen leyes mentales estrictas a partir de las cuales puedan explicarse y predecirse acaecimientos mentales.

Comencemos analizando la tesis del monismo físico. Es importante tener en cuenta que dicha tesis hace referencia no a acaecimientos tipo, sino a ejemplares, o acaecimientos "token", es decir, a entidades particulares e irrepetibles que ocupan una zona espaciotemporal determinada y que, según Davidson, son el tipo de entidades propiamente vinculadas por la relación causal. Sin entrar en difíciles problemas metafísicos que nos desviarían de nuestros propósitos, podemos pensar en los acaecimientos como aquel tipo de entidades a la que refieren expresiones como 'el regreso de Elián González a Cuba', 'el hundimiento del Titanic', o 'la detención de Pinochet en Londres'. Estos acaecimientos particulares, y esto es algo esencial al modo como 
Davidson los entiende, pueden ser descritos de modos diversos, es decir, en general, más de una descripción convendrá al mismo acaecimiento. Por ejemplo, puedo referirme a la detención de Pinochet como el acaecimiento que enfrió las relaciones diplomáticas entre España y Chile, o al hundimiento del Titanic como el mayor naufragio de la marina civil anglosajona.

Según Davidson, un acaecimiento es mental si podemos describirlo usando verbos de actitud proposicional, esto es, verbos como 'desear', 'creer', 'pretender', o sustantivaciones de los mismos como 'la creencia de Juan de que...', 'el deseo de Luisa de que... ', etc. El mismo Davidson reconoce que este criterio está lejos de ser satisfactorio, pues de él se desprende que cualquier acaecimiento es mental. En efecto, parece que siempre podremos describir un acaecimiento utilizando un verbo de actitud proposicional (por ejemplo, puedo referirme a un acaecimiento paradigmáticamente no mental como el derrumbamiento del edificio de enfrente de mi casa con la descripción 'el acaecimiento que sumió a mi vecina en una profunda depresión'). Con todo, es éste un criterio que nos permite recoger los acaecimientos genuinamente mentales. Por otro lado, un acaecimento físico será aquel que pueda describirse sin utilizar verbos de actitud proposicional o sustantivaciones de los mismos. Esto bastará a Davidson.

Davidson evita hablar de descripciones canónicas de acaecimientos o de propiedades constitutivas de los mismos a las que podrían hacer referencia dichas descripciones canónicas, como un modo razonable de evitar el resultado nada intuitivo de que todo acaecimento es mental. Sin duda, con ello, su monismo anómalo ganaría en claridad y precisión. Pero, en todo caso, no son éstos los problemas que nos interesan en el presente trabajo y bastará en lo sucesivo con que tengamos a la vista el criterio de Davidson al hablar de acaecimientos mentales.

Resumiendo, pues, la tesis del monismo físico (MF) dice que todos los acaecimientos denotados por descripciones del tipo de 'la creencia de Pablo de que Pinochet fue detenido en Londres' o 'el deseo de Carmen de volver a México por vacaciones' pueden también ser denotados por descripciones que no contengan términos psicológicos de ningún tipo. 
Por lo que respecta a la tesis (AM) conviene subrayar que Davidson tiene lo que podríamos llamar una concepción lingüística de las leyes. Entiende por ley un enunciado universal con ciertas características epistémicas, a saber, que sustenta contrafácticos y que se ve confirmado por sus instancias positivas. ${ }^{2}$ A los enunciados universales que no tienen estas características los llama generalizaciones, opuestos a las leyes. Así, por ejemplo, 'todas las esmeraldas son verdes' es una ley; sin embargo, 'todas las esmeraldas son verzules' es una generalización, pues el hecho de dar con una esmeralda verde aumenta nuestra confianza en la verdad del primer enunciado, pero el hecho de dar con una esmeralda verzul no aumenta nuestra confianza en la verdad del segundo. ${ }^{3}$

A continuación introduciré una distinción terminológica que nos será útil más adelante. Según esta concepción lingüística de las leyes, diremos que un acaecimiento queda subsumido bajo una ley si puede describírsele correctamente con el vocabulario de la ley. Nótese que si no utilizamos la descripción correcta no podemos inferir por ello que no quede efectivamente subsumido por dicha ley. Un ejemplo aclarará lo que queremos decir. Supongamos que es una ley el enunciado siguiente: "todos los resfriados causan un descenso del nivel de atención’. Así, el acaecimiento consistente en la afección que Carlos contrajo en su viaje a Noruega, así descrito, no se ve que quede subsumido o cubierto por la ley que relaciona el resfriado con la atención. Pero de ello no se sigue que dicho acaecimiento no quede efectivamente subsumido por la ley; esto último dependerá de si puede ser descrito de modo correcto como un resfriado. Diremos, entonces, que un acaecimento se muestra subsumido bajo una ley

2 Davidson 1970, p. 217.

${ }^{3}$ Goodman 1955. Con todo, tal vez valga la pena matizar que Davidson no considera que 'verzul' sea un predicado no proyectable. Lo único que ocurre, según él, es que no casa bien con el término 'esmeralda' para formar una ley. En cambio sí casa bien, por ejemplo, con el término 'esmerafiro'; término que definimos de tal modo que se aplique a esmeraldas o a zafiros en ciertas condiciones. De un modo análogo, dirá Davidson, tampoco los predicados físicos y los predicados mentales casan bien para formar leyes, por lo que no podrá haber leyes psicofísicas (véase Davidson 1970, p. 218). Sobre esto último, véase más adelante. 
cuando aparece descrito correctamente con el vocabulario de la ley.

Por otro lado, una ley estricta, según Davidson, es aquella que puede ser refinada hasta eliminar todas sus excepciones sin salir del tipo de vocabulario en el que la ley se formula originalmente, es decir, sin recurrir a conceptos ajenos a aquellos expresados por el vocabulario en el que se formula la ley originalmente. Davidson declara que sólo las leyes físicas son estrictas, y en particular no lo son las leyes químicas o biológicas. Sólo la física proporciona un marco conceptual, en palabras del autor, exhaustivo y cerrado, esto es, un marco en el que cualquier fenómeno captado por un concepto físico puede ser explicado de un modo completo a través de leyes en las que aparezcan únicamente conceptos físicos. Recapitulando, pues, podemos decir que la tesis del anomalismo mental sostiene que no hay leyes estrictas formuladas con términos que expresen conceptos mentales.

\section{El argumento a favor del anomalismo mental}

Una vez presentadas las tesis del monismo anómalo, iniciemos el análisis de los argumentos que Davidson ofrece para sustentarlas. Comenzaremos por la segunda tesis, pues, como veremos, constituye una de las premisas del argumento a favor de la primera. La argumentación de Davidson responde al siguiente esquema:

\section{Argumento 1}

1.1 De haber leyes estrictas que involucren conceptos mentales, o son leyes estrictas psicológicas (esto es, leyes que involucran sólo conceptos mentales) o son leyes estrictas psicofísicas (esto es, leyes que involucran conceptos mentales y conceptos físicos). 1.2 No hay leyes psicológicas estrictas.

1.3 No existen leyes estrictas psicofísicas.

Anomalismo mental

Davidson no discute la premisa 1.1, pero resulta obvia dada la caracterización de lo mental y lo físico que supone y que hemos

4 Davidson 1970, p. 218. 
discutido en la sección anterior. Por lo que respecta a la premisa 1.2, la justificación que ofrece Davidson se limita a la observación de que son muchos los fenómenos de carácter no mental que afectan lo mental como para poder pensar que los conceptos psicológicos constituyen un marco conceptual exhaustivo y cerrado y que en un futuro dispondremos de leyes psicológicas estrictas. 5 Creo que también podemos dar la razón a Davidson en este punto, pues ciertamente cualquier vinculación nómica entre los estados mentales de una persona viene garantizada por cierta condición física, e igualmente, cualquier vinculación entre los estados mentales de alguien y sus acciones exige también el cumplimiento de ciertas condiciones físicas en el medio externo a la persona.

Ahora bien, el argumento en favor de la premisa 1.3 es notoriamente complejo e intrincado y de hecho ha suscitado una gran polémica entre los intérpretes de Davidson. A continuación se ofrecerá una reconstrucción del mismo que trata de ser fiel a los textos, por desgracia escasos y poco claros. Dicha reconstrucción reflejará nuestro grado de comprensión del argumento, de la que no nos sentimos totalmente satisfechos. Con todo, y a pesar de no estar en condiciones de asegurar que la reconstrucción que va a ofrecerse reproduzca perfectamente el pensamiento del autor, sí estamos convencidos de que, al menos en lo que respecta a los elementos esenciales del argumento que entrarán en conflicto con la tesis T, y que por ello nos interesan especialmente en este trabajo, la reconstrucción es correcta.

Parte del problema de interpretación que presenta el argumento radica en su originalidad. En efecto, la mayoría de los filósofos que han argumentado contra la existencia de leyes psicofísicas, o también contra la posibilidad de explicar o reducir los acaecimientos mentales a acaecimientos físicos, han tomado como punto de partida el llamado "punto de vista de la primera persona", es decir, el fenómeno de autoatribución de acaecimientos mentales, o bien los llamados "acaecimientos fenoménicos"; por ejemplo, dolores, emociones o sensaciones visuales. ${ }^{6}$ Frente

5 Ibid., p. 224.

6 Véanse Nagel 1974, Kripke 1981, Jackson 1986. 
a esto, el argumento de Davidson parte del carácter intencional de acaecimientos mentales como creencias, deseos o intenciones (téngase en cuenta la caracterización de lo mental discutida en la sección 1) y del punto de vista de tercera persona, esto es, la actividad consistente en la atribución de acaecimientos mentales a terceros.

El argumento de Davidson se articula en dos premisas:

Argumento 1.3, a favor de que no hay leyes estrictas psicofísicas. 7

1.31 Hay algo constitutivo de nuestros conceptos mentales, cuando los utilizamos para atribuir acaecimientos mentales a otros, que no es constitutivo de nuestros conceptos físicos, es decir, de aquellos conceptos expresados en nuestras leyes físicas.

1.32 La diferencia entre los conceptos mentales y los conceptos físicos aludida en 1.31 imposibilita la existencia de leyes psicofísicas, esto es, la existencia de enunciados universales donde se mezclan predicados mentales y predicados físicos con las características epistémicas pertinentes.

A continuación trataremos de aclarar hasta donde podamos este argumento 1.3. En primer lugar, por lo que respecta a 1.31, la característica de nuestros conceptos mentales en la que quiere hacerse hincapié es la siguiente. Según Davidson, la atribución de un acaecimiento mental $m$ a un agente $A$ viene regulada por una condición de racionalidad que exige que $m$ mantenga unas relaciones de coherencia con el resto de acaecimientos mentales atribuidos a $A$, de tal modo que $A$, mediante tales atribuciones, se nos aparezca como un ser racional. ${ }^{8}$

La necesidad de este requisito de racionalidad que debe gobernar, según Davidson, la práctica de atribuir acaecimientos mentales a terceros, se comprende mejor si tenemos presente el punto de vista de tercera persona desde el cual Davidson analiza siempre los conceptos mentales. La idea fundamental es que lo

7 Davidson 1970, p. 222.

8 Ibid., pp. 222-223. 
que nos empuja a atribuir estados mentales a terceros es comprender sus acciones. Lo que pretendemos es que la conducta del agente se nos aparezca precisamente como algo racional e inteligible. Ésta sería la función básica de tales conceptos, por eso el requisito de racionalidad es constitutivo de los mismos. Ahora bien, la racionalización de la conducta de un agente no se consigue atribuyéndole un único acaecimiento mental aislado, sino que llevar tal práctica a buen fin exige atribuir un conjunto (tal vez sería más preciso decir un sistema) de acaecimientos mentales a ese agente, los cuales, en virtud de su contenido, mantengan entre ellos una relación de coherencia tal que la conducta del agente quede debidamente racionalizada. Por ejemplo, no podemos racionalizar la conducta de un agente consistente en proferir un ejemplar de la oración 'hace frío' limitándonos a atribuirle la creencia de que hace frío. Debemos, además, si queremos que la conducta quede debidamente racionalizada, atribuirle el deseo de transmitirnos tal creencia y también la creencia de que profiriendo un ejemplar de la oración 'hace frío' conseguirá transmitírnosla (amén de otras creencias, como la de que sus interlocutores entienden el español, etc.).

De hecho, Davidson va algo más lejos y defiende la tesis de que el contenido de un acaecimiento mental depende de las relaciones de coherencia que mantiene con el resto de los estados mentales que constituyen el sistema atribuido al agente. Así pues, Davidson suscribe, decididamente, la tesis del holismo del contenido mental. ${ }^{9}$

Si no estoy equivocado, estas ideas, que pretenden justificar la premisa 1.31, vienen recogidas en el siguiente texto:

No se asignan creencias a alguien una por una a partir de su conducta verbal, de sus decisiones o de otros signos locales por muy claros y evidentes que resulten, pues damos sentido a creencias particulares sólo en la medida en que son coherentes con otras creencias, preferencias, intenciones, esperanzas, miedos, expectati-

9 Davidson 1970, p. 221. 
vas y demás [...] el contenido de una actitud proposicional deriva del lugar que ocupa en el sistema. ${ }^{10}$

Así pues, podemos reformular la premisa 1.31 del argumento 1.3 del siguiente modo: es constitutivo de nuestros conceptos mentales que la atribución de un acaecimiento mental $m$ a un agente $A$ no tiene ningún sentido excepto en el transfondo de la atribución de todo un sistema $S$ de acaecimientos mentales a $A$ con los cuales $m$ mantenga relaciones de coherencia, de las que deriva el contenido de $m$, de tal modo que al atribuir $S$ a $A$, éste y su conducta devengan racionales.

De este modo, el requisito de racionalidad nos impedirá atribuir un acaecimiento mental a un agente $A$ si el primero no guarda las relaciones de coherencia prescriptivas con el sistema de acaecimientos mentales que hemos atribuido a $A$ con el fin de racionalizar su conducta. Davidson admite que en algunos casos podemos no ser capaces de atribuir un sistema de acaecimientos que racionalice plenamente una conducta determinada de un agente, y advierte que tal posibilidad es compatible con el requisio de racionalidad. Ahora bien, si no somos capaces de atribuir al agente un sistema de acaecimientos mentales que racionalice mínimamente su conducta, entonces, según se desprende del requisito de racionalidad, es conceptualmente imposible atribuir acaecimientos mentales a ese sujeto. Por tanto, y éste es un punto clave en la argumentación de Davidson, el requisito de racionalidad constituye una condición a priori, obtenida por razones puramente conceptuales, en la atribución de cualquier acaecimiento mental. Ello se obtiene inmediatamente del hecho de considerar tal requisito condición de aplicación de los conceptos mentales, tesis que, como avanzamos antes, deriva a su vez de considerar que está en la naturaleza de los conceptos mentales el hecho de usarlos para racionalizar la conducta de terceros.

10 Id., la traducción es mía. Texto original: "there is no assigning of beliefs to a person one by one on the basis of his verbal behaviour, his choices, or other local signs no matter how plain and evident, for we make sense of particular beliefs only as they cohere with other beliefs, with preferences, with intentions, hopes, fears, expectations and the rest [...] the content of a propositional attitude derives from its place in the pattern." 
La justificación de la premisa 1.31 concluye con la observación, a todas luces obvia, de que la aplicación de conceptos físicos no parece estar regulada por el requisito de racionalidad.

La premisa 1.32 del argumento 1.3 dice, como se anticipó, que esta diferencia en el modo como funcionan los conceptos mentales y los conceptos físicos imposibilita la existencia de leyes psicofísicas estrictas. Davidson dice que el caso de las supuestas leyes psicofísicas es parecido al de enunciados como 'todas las esmeraldas son verzules', que no son proyectables, esto es, no se ven confirmados por sus instancias positivas, con lo cual, según el criterio de Davidson, no pueden ser leyes. La razón de ello para Davidson, como se dijo anteriormente, ${ }^{11}$ estriba en el hecho de que el predicado 'verzul' no casa bien con el predicado 'ser una esmeralda', pero no en el hecho de que el predicado 'verzul' no sea proyectable. Sí lo es, y de hecho casa bien con predicados como 'ser un esmerafiro', por lo que el enunciado 'todos los esmerafiros son verzules' es proyectable y debe, por lo que respecta a este requisito, considerarse una ley. ${ }^{12} \mathrm{De}$ modo análogo, los predicados mentales no casan bien con los predicados físicos para formar enunciados proyectables. Tal fenómeno se debe, por supuesto, al requisito de racionalidad, el cual, como vimos, gobierna los primeros pero no los segundos. Sin embargo, el propio Davidson reconoce que la simple analogía con los enunciados tipo 'todas las esmeraldas son verzules' no basta para justificar la premisa 1.32, pues no nos parece que los enunciados psicofísicos (esto es, enunciados que contienen predicados mentales y predicados físicos) no sean proyectables, como sí nos lo parece en el caso de enunciados como 'todas las esmeraldas son verzules'. ${ }^{13}$

El problema de interpretación del argumento de Davidson es que no queda claro cuál es la justificación adicional de esta

11 Véase la nota 3.

12 Véase Davidson 1966. La posición de Davidson abre la posibilidad de que un enunciado no sea proyectable aunque todos los predicados de que conste lo sean. 'Todas las esmeraldas son verzules' sería un ejemplo de un enunciado tal.

13 Davidson 1970, pp. 218-219. 
premisa. A nuestro juicio, uno de los pocos textos en los que el autor se esfuerza por aclarar cuál sea esta justificación adicional, y que nos servirá para terminar nuestra reconstrucción del argumento 1.3, es el siguiente:

¿Al declarar que no existen leyes (estrictas) psicofísicas, no estaremos cazando en el coto empírico reservado a la ciencia? [...] Si el caso de las supuestas leyes que vinculan lo mental y lo físico es distinto, ello puede deberse solamente al hecho de que permitir la posibilidad de tales leyes supondría un cambio de tema. Por un cambio de tema entiendo aquí: decidir no aceptar el criterio de lo mental en términos del vocabulario de las actitudes proposicionales. ${ }^{14}$

Mi interpretación de este texto es la siguiente: Son razones conceptuales, puramente a priori, las que descartan la posibilidad de la existencia de leyes psicofísicas. Ahora bien, aseverar o negar la existencia de una ley natural de cualquier tipo (es decir, de una ley no lógica o conceptual) parece algo paradigmáticamente a posteriori, y por ello, una pieza que sólo puede cobrar el investigador empírico. Entonces, ¿cómo es posible que razones puramente a priori nos permitan descartar la existencia de ciertas posibles leyes naturales? Davidson nos dice en este texto que el caso de las pretendidas leyes psicofísicas constituye una excepción. Lo es porque hay algo constitutivo de nuestros conceptos mentales, a saber, el requisito de racionalidad, que no es constitutivo de nuestros conceptos físicos y que hace conceptualmente imposible la existencia de tales leyes. Hasta aquí no hay más que una reiteración de las premisas del argumento 1.3, todavía queda pendiente una justificación. Y la justificación (la única justificación que hemos sido capaces de encontrar rastreando los escritos de Davidson) es que suponer la existencia de

${ }^{14}$ Ibid., p. 216, la traducción es mía. Texto original: "Do we, by declaring that there are no (strict) psychophysical laws, poach on the empirical preserves of science? [...] If the case of supposed laws linking the mental and the physical is different, it can only be because to allow the possibility of such laws would amount to changing the subject. By changing the subject I mean here: deciding not to accept the criterion of the mental in terms of the vocabulary of the propositional attitudes." 
tales leyes conlleva un "cambio de tema". Y esto parece querer decir que conllevaría la posibilidad de atribuir un acaecimiento mental sin tener en cuenta el requisito de racionalidad, lo cual es absurdo, pues, como hemos visto, al ser constitutivo de los conceptos mentales, tal requisito es una condición a priori de la atribución de acaecimientos mentales.

A mi juicio, J. Kim ha ilustrado mejor que nadie en qué consistiría el "cambio de tema" que vendría propiciado por la existencia de una ley psicofísica. ${ }^{15}$ Supongamos por un momento que fuera una ley estricta el enunciado 'la ocurrencia de una acaecimiento físico $F$ es suficiente para la ocurrencia de un acaecimiento mental tipo $M^{\prime}$. Si existiera una tal ley, entonces la atribución a un agente de un acaecimiento físico de tipo $F$ sería un requisito suficiente para la atribución a ese agente de un acaecimiento mental de tipo $M$. Pero en este caso, nuestra atribución de acaecimientos mentales tipo $M$ no vendría gobernada por el requisito de racionalidad; dicho de otro modo: en caso de que existiera la ley psicofísica del ejemplo, podríamos hacer atribuciones de cierto tipo de acaecimientos mentales sin observar el preceptivo requisito de racionalidad.

Lo que creo que debe añadirse a la explicación de Kim para completarla es lo siguiente. No observar el requisito de racionalidad en este caso supondría un cambio de tema, porque al usar un concepto $M$ no regulado por el requisito de racionalidad, y al ser tal requisito constitutivo de los conceptos mentales, no podríamos considerar el predicado "ser $M$ " como un predicado mental. Por ello, la existencia de leyes psicofísicas, que mezclen conceptos mentales y físicos, es conceptualmente imposible.

Igualmente, la existencia de una ley psicofísica como la del ejemplo permitiría atribuir un acaecimiento mental de cierto tipo y, por tanto, con un contenido definido, sin tener en cuenta las relaciones que ese acaecimiento mental pueda guardar con otros que podamos atribuir, vulnerando el principio del holismo mental según el cual, recordemos, el contenido de un acaecimiento mental deriva del lugar que ocupa en el sistema formado por el conjunto de acaecimientos mentales atribuidos al agente y sus

15 Kim 1985. 
relaciones de coherencia. Todo ello mostraría en definitiva que por razones puramente conceptuales, y como excepción, podemos descartar la existencia de cierto tipo de leyes. Suponer la existencia de leyes psicofísicas, digámoslo una vez más, es conceptualmente imposible (si bien imaginamos que Davidson no tendría reparos en reconocer que se trata de una imposibilidad conceptual que no está en la superficie, sino que requiere argumentación; es decir, no sería el tipo de imposibilidad conceptual que conlleva suponer círculos cuadrados, sino, por ejemplo, la que supone imaginar viajes en el tiempo pasado).

Hay un modo que considero más fielmente davidsoniano de explicar el punto crucial en la justificación de la premisa 1.32. Tomemos de nuevo el enunciado 'la ocurrencia de un acaecimiento físico tipo $F$ es suficiente para la ocurrencia de un acaecimiento mental tipo $M^{\prime}$. Por razones conceptuales podemos decir que este enunciado no es proyectable, es decir, que no se ve confirmado por sus instancias positivas. Véamoslo. Sea $i$ una instancia positiva del enunciado, es decir, un caso en el que atribuimos a un agente un acaecimiento físico tipo $F$ y un acaecimiento mental tipo $M$. Pues bien, la existencia de un caso así no nos hace esperar que la siguiente atribución a un agente de un acaecimiento tipo $F$ comportará la atribución a este mismo agente de un acaecimiento tipo $M$, pues la atribución de acaecimientos tipo $M$ depende conceptualmente del requisito de racionalidad del cual no depende la atribución de un acaecimiento tipo $F$. La atribución de un acaecimiento mental tipo $M$ depende de la atribución de una serie de acaecimientos mentales con los cuales mantenga relaciones de coherencia que permitan racionalizar la conducta del sujeto, y para nada de aquello de lo que pueda depender la atribución de un acaecimiento físico tipo $F$.

Resumamos, pues, las razones de Davidson en favor de la premisa 1.32. En virtud de la diferencia entre los requisitos conceptuales que involucra la atribución de acaecimientos mentales por un lado y de acaecimientos físicos por otro, la existencia de leyes psicofísicas es conceptualmente imposible, pues supondría una violación del requisito de racionalidad que regula la atribución de cualquier acaecimiento mental. 
Ahora bien, si el criterio de racionalidad que regula la atribución de acaecimientos mentales no tiene una contrapartida o una correspondencia en el modo de funcionar de nuestros conceptos físicos, entonces resulta cuando menos sorprendente sostener la tesis del monismo físico. De hecho, la sorpresa aumenta si tenemos en cuenta la observación de Davidson de que el anomalismo mental remite al fenómeno quineano de la indeterminación de la traducción. ${ }^{16}$ Interpreto esta referencia como una advertencia de la posibilidad de que exista en un caso concreto más de un sistema de acaecimientos mentales, no equivalentes, que podamos atribuir a un agente para racionalizar su conducta. Por ejemplo, podemos racionalizar una proferencia de Juanito de un ejemplar de 'hace frío' atribuyendo a Juanito (entre otros acaecimientos mentales) la creencia de que hace frío, el deseo de transmitírnosla y la creencia de que profiriendo un ejemplar de 'hace frío' satisfará su deseo; pero también podemos racionalizar la conducta de Juanito atribuyéndole el deseo de hacernos creer lo contrario de lo que piensa, y las creencias de que hace calor y de que profiriendo un ejemplar de 'hace frío' conseguirá engañarnos, entre otras. Parece que la idea de Davidson es que la opción entre sistemas o "manuales de racionalización" distintos e incompatibles se mantiene aun cuando tomemos en consideración la conducta global del agente, y no únicamente un comportamiento aislado. El problema adicional que plantea la indeterminación es que los acaecimientos mentales atribuidos en cada sistema posible responderán a las exigencias del requisito de racionalidad, atribuyéndose en cada caso, según el manual de racionalización que se escoja, acaecimientos mentales distintos con un contenido distinto, sin que haya modo de decidir, ni de hecho ninguna razón de principio para decidir, entre estas alternativas. Esa clase de indeterminación que surge del requisito de racionalidad está, por supuesto, ausente en el caso de la atribución de acaecimientos físicos. ¿Cómo esperar, entonces, que cada acaecimiento mental atribuido sea, de hecho, un acaecimiento físico?

Así las cosas, el argumento davidsoniano en favor del anomalismo mental, basado en el carácter constitutivo del requisito de

16 Davidson 1970, p. 222. 
racionalidad para los conceptos mentales, parece cuando menos una seria amenaza para la tesis del monismo físico. Ahora bien, Davidson está convencido de que dispone de un argumento independiente y concluyente en favor de esta última tesis. Nuestro propósito es mostrar que este argumento, que analizaremos en la sección siguiente, parece incompatible con el argumento y la tesis misma del anomalismo mental, y no meramente independiente de él.

\section{El argumento en favor del monismo físico: nuestro problema}

Afortunadamente este argumento está formulado con mayor claridad. Se obtiene de las siguientes tres premisas:

Argumento 2, en favor del monismo físico: ${ }^{17}$

2.1 Los acaecimientos mentales interaccionan causalmente con acaecimientos físicos.

2.2 Principio del Carácter Nomológico de la Causalidad (PCNC): si un enunciado del tipo ' $e$ causa $e^{\prime}$ 'es verdadero, entonces existen descripciones de $e$ y $e^{\prime}$ mediante las cuales $e$ y $e^{\prime}$ se muestran subsumidos bajo una ley causal estricta.

2.3 Anomalismo mental.

A simple vista puede parecer que estas premisas son incompatibles, pero Davidson se esfuerza en hacernos ver que no es éste el caso. En primer lugar, hay que tener presente otra vez que en la premisa 2.1 se hace referencia a acaecimientos "token" y no a tipos de acaecimientos. Todo lo que se afirma en ella, así pues, es que hay relaciones causales entre acaecimientos mentales particulares y acaecimientos físicos particulares. Además, hay que traer a la mente la distinción crucial que Davidson propone y defiende entre la relación causal y la relación de explicación causal. ${ }^{18}$ La primera es una relación extensional entre acaecimientos, relación que mantienen, por lo tanto, con independencia de como se los describa. La segunda, en cambio, es una relación intensional, se mantiene entre dos acaecimientos sólo cuando

17 Davidson 1974, p. 231.

18 Véase Davidson 1967. 
ambos son descritos de tal modo que se muestran subsumidos bajo una ley causal estricta. Lo que afirma, pues, la premisa 2.2, el PCNC, es precisamente que se produce una relación causal entre acaecimientos sólo en el caso de que existan descripciones de tales acaecimientos a través de las cuales se muestren subsumidos bajo una ley causal estricta; o dicho de otro modo, que dos acaecimientos particulares están relacionados causalmente sólo cuando existen descripciones de los mismos a través de las cuales uno, el acaecimiento-causa, explique causalmente el otro, el acaecimiento-efecto.

Hechas estas aclaraciones, el argumento de Davidson puede reconstruirse como sigue. Sea $m$ un acaecimiento mental que interacciona causalmente con un acaecimiento físico $f$, de acuerdo con la premisa 2.1. Según el PCNC, premisa 2.2, tiene que haber descripciones de $m$ y $f$ mediante las cuales $m$ y $f$ se muestren subsumidos bajo una ley causal estricta. Ahora bien, dado el anomalismo mental, premisa 2.3, tal ley causal estricta únicamente puede ser física. Por tanto, debe haber una descripción física de $m$; así pues, $m$ es un acaecimiento físico. ${ }^{19}$

Antes de analizar este argumento y el lugar donde a nuestro juicio el razonamiento de Davidson se vuelve inconsistente, quizás convenga hacer notar que el monismo anómalo está lejos de dar una respuesta satisfactoria a la tercera antinomia kantiana, como parece creer su defensor. La supuesta solución a la antinomia estribaría, al parecer, en la distinción entre causalidad y explicación causal. Por un lado, tenemos que los acaecimientos físicos se muestran, a través de sus descripciones físicas, subsumidos bajo leyes físicas estrictas; pero, por otro lado, resulta que al no haber leyes psicológicas ni psicofísicas el ámbito de lo mental, descrito como tal, no aparece regulado por leyes, quedando así, en este ámbito, un margen

19 Nótese que las observaciones hechas en la sección 1 acerca del carácter poco satisfactorio del criterio davidsoniano de lo físico no debilitan la conclusión de este argumento. El razonamiento permite en efecto concluir que existe una descripción de $m$ que lo muestra subsumido bajo una ley física estricta, y eso descarta posibles descripciones físicas espurias de $m$ que amenazarían la conclusión de que $m$ sea después de todo un acaecimiento físico. 
para la libertad humana. Pero esta solución dista, por supuesto, de ser una solución satisfactoria. En realidad, de acuerdo con la tesis del monismo físico, lo que ocurre es que los acaecimientos mentales responsables causales de nuestras acciones son acaecimientos físicos que causan estas acciones obedeciendo las leyes causales estrictas de la naturaleza. Siendo esto así, ciertamente no se ve qué alivio pueda representar la doctrina del monismo anómalo para el filósofo preocupado por la tercera antinomia de la razón, pues es parte de tal doctrina que el hecho de que no existan leyes psicológicas ni psicofísicas no es óbice para que la relación causal entre nuestras razones para actuar y nuestras acciones intencionadas no siga leyes estrictas.

Pero el problema en el razonamiento de Davidson que es objeto de este trabajo no tiene que ver con la tercera antinomia. El problema que queremos resaltar es que la justificación de este argumento 2 entra en conflicto con las conclusiones del argumento 1. Para ver por qué esto es así, pasemos a analizar el argumento 2. La tercera premisa, 2.3, ha sido discutida suficientemente en la sección anterior, y la segunda, el PCNC, Davidson la acepta sin argumentarla. También la aceptaremos nosotros aquí porque la inconsistencia que queremos poner de manifiesto no tiene que ver con ella. ${ }^{20} \mathrm{La}$ inconsistencia tiene que ver con la justificación de la premisa 2.1. Davidson la justifica apelando precisamente a la tesis $\mathrm{T}$, mencionada al principio de este trabajo. La idea es que al menos ciertos acaecimientos mentales y ciertos acaecimientos físicos interaccionan causalmente (Davidson reconoce que, en rigor, la tesis del monismo físico debería ceñirse únicamente a este cierto tipo de acaecimientos mentales); a saber, aquellos que figuran como razones para actuar en racionalizaciones correctas de la conducta de un agente. La razón para ello es que tales razones constituirán una racionalización correcta de la conducta, ofrecerán una buena explicación de ella, sólo cuando sean causas de tal conducta, de acuerdo, pues, con la tesis $\mathrm{T}$.

Con la defensa de la tesis T, Davidson se oponía a una influyente corriente wittgensteiniana que opinaba que el vínculo

20 No obstante, véase la sección final, donde se discutirá el PCNC. 
explicativo entre razones y acciones no era causal, sino racionalizador. Según este punto de vista, las razones - acaecimientos mentales como creencias o deseos - que atribuimos a un agente con tal de racionalizar su conducta explican un comportamiento suyo únicamente en la medida en que nos la hacen inteligible, comprensible o propia de un ser racional, y en absoluto porque constituyan las causas (o algunas de las causas) de tal conducta. Una opinión del mismo tenor se retrotrae a los escritos de Dilthey y a su famosa distinción entre explicación y comprensión ("verstehen"), en la cual se funda la tajante distinción que este autor propugnó entre ciencias humanas o sociales por un lado, y ciencias naturales por el otro, y que hizo fortuna.

Davidson se opuso a estas opiniones defendiendo la tesis $\mathrm{T}$. De ahí la importancia que debe atribuirse a dicha tesis desde un punto de vista histórico. La defensa de la tesis T que presentó Davidson en su trabajo "Actions, Reasons and Causes" se basó en una lúcida observación que con toda justicia no ha sido pasada por alto por los teóricos de la acción desde que fue formulada. La observación es simplemente que aún en el caso de que los acaecimientos mentales que atribuyamos a un agente para comprender su acción nos la hagan inteligible, no consideraremos tal atribución una explicación correcta de dicha acción a menos que consideremos que los acaecimientos mentales atribuidos sean causas, en el sentido usual del término, de la propia acción. ${ }^{21}$ Así, Edipo tiene ciertamente una serie de razones para matar a su padre, razones que nos hacen inteligible su acción de asesinarlo, pero claramente no son estas razones las que explican el asesinato, pues Edipo no asesina en realidad a su padre a causa de estas razones. ${ }^{22}$

Pongamos un nuevo ejemplo para ilustrar mejor este punto crucial. Santiago Salvador fue un anarquista catalán que arrojó dos bombas sobre el patio de butacas del Liceo de Barcelona la

${ }^{21}$ Para que la explicación sea considerada correcta es necesario además suponer que la cadena causal que une razones con acciones es normal. Soslayo el espinoso problema de determinar qué tipo de cadenas causales son normales pues no afecta para nada a los problemas que se discuten en este trabajo.

22 Davidson 1974, p. 232. 
noche del 7 de noviembre de 1893. Salvador, al parecer, quería cometer el atentado convencido de que tal hecho serviría a la estrategia anarquista radical de desmoralizar y amedrentar a la alta burguesía catalana. Éstas parece que fueron sus razones para actuar. Llegado el momento que juzgó más oportuno arrojó las bombas provocando una horrible masacre. Pues bien, en este caso diríamos, sin dudarlo, que las razones de Salvador racionalizan y explican perfectamente su acción atroz. Pero imaginemos ahora una situación contrafáctica en la cual Salvador tiene las mismas razones que antes, las mismas ganas de servir a la estrategia anarquista radical y la misma convicción acerca de la utilidad de su atentado; pero en este caso, cuando se dispone a arrojar las bombas, sufre un ataque epiléptico y a causa de las convulsiones de su cuerpo arroja involuntariamente las bombas sobre la platea del teatro. Davidson opina, y en mi opinión tiene toda la razón, que en esta situación contrafáctica no diríamos que las razones de Salvador explican su acción, a pesar de que la racionalizan, la hacen tan comprensible como en la situación anterior, y que no lo diríamos porque en este caso contrafáctico a partir de la información que se nos ha dado no consideramos tales razones causa de su acción. La lección que se extrae de estos ejemplos es, precisamente, la validez de la tesis $\mathrm{T}$ : no juzgamos explicativo el vínculo entre razones y acciones a menos que juzguemos que existe entre ambas un vínculo causal.

Lo que a nuestro juicio muestra el razonamiento de Davidson, y que nos parece de la mayor importancia, es que por racionalización de una acción A entendemos explicación de A en la cual se apela a las causas mentales de A. La defensa de la tesis T, a nuestro juicio impecable, que lleva a cabo Davidson ciertamente desmiente a los filósofos que juzgan compatible afirmar que las acciones pueden ser racionalizadas, y con ello explicadas, a través de acaecimientos mentales y que no existen vínculos causales entre acaecimientos mentales y acciones, entendidas estas últimas como acaecimientos físicos. Sin embargo, no garantiza, como parece creer el propio Davidson, la verdad de la premisa 2.1, pues todas las racionalizaciones podrían ser erróneas; es decir, podría disponerse de un argumento independiente que mostrase 
que los acaecimientos mentales carecen de eficacia causal compatible con la tesis $\mathrm{T}$ y la defensa que Davidson ha hecho de ella. Dejaremos por el momento en suspenso esta posibilidad, de la que diremos algo más en la sección final, y a continuación abordaremos el objetivo central de este trabajo: mostrar que la defensa que Davidson hace de la tesis $\mathrm{T}$ es incompatible con su defensa del anomalismo mental.

Insistimos en que, a nuestro parecer, la defensa de Davidson de la tesis $\mathrm{T}$ resulta correcta y que, además, es de la mayor importancia por las razones que hemos ido desgranando en párrafos anteriores. El problema que queremos señalar es que, a nuestro juicio, dicha defensa resulta incompatible con la tesis del anomalismo mental, y de ahí la inconsistencia que venimos anunciando desde el principio del presente trabajo. Veamos. Según el argumento de Davidson, las razones son explicativas de una acción sólo si la causan, o dicho con mayor rigor, sólo consideraremos explicativa de una acción la apelación a acaecimientos mentales del agente si consideramos tales acaecimientos como causas. Ahora bien, se sigue de la tesis del monismo anómalo que las racionalizaciones no pueden ser explicaciones causales. No pueden serlo porque en una racionalización nos referimos a acaecimientos mentales mediante descripciones mentales y, al no haber leyes psicológicas ni psicofísicas, es imposible que en una racionalización tales acaecimientos se muestren subsumidos bajo alguna ley causal estricta.

Entre razones descritas como tales, es decir, en términos mentales, y acciones puede verificarse a lo sumo una relación causal (que, como se recordará, es un tipo de relación extensional entre acaecimientos) pero no puede verificarse una relación de explicación causal; tal hecho exigiría lo que la tesis del anomalismo mental niega, a saber: que hubiera leyes psicofísicas o psicológicas. Sin embargo, la tesis $\mathrm{T}$ nos dice que la mención de razones la consideramos explicativa de una acción sólo si consideramos tales razones causas de la acción. Ahora bien, al no mostrarse las razones subsumidas bajo ninguna ley causal con la acción que racionalizan, no hay razón para esperar que verifiquen una relación causal con esa acción. No hay ningún motivo para creer que haya descripciones de esas razones mediante las cuales tales 
razones se muestren vinculadas a través de una ley causal a la acción. Y en ausencia de motivos, queda socavada la verosimilitud de la premisa 2.1.

Un ejemplo ayudará a aclarar la dificultad que queremos señalar. Consideremos el enunciado 'el huracán Mitch causó la muerte de miles de personas en Centroamérica hace dos años'. Podemos decir que este enunciado constituye una explicación causal de la muerte de miles de personas en Centroamérica hace dos años porque pensamos que existe una ley causal que vincula acaecimientos consistentes en huracanes de cierta intensidad con acaecimientos consistentes en la muerte de miles de personas. En el enunciado nos referimos a esos acaecimientos mediante descripciones que muestran que están subsumidos bajo esta ley causal. Supongamos ahora que el huracán Mitch fue el acaecimiento que más ha impresionado a Juanito y que la muerte de miles de personas en Centroamérica hace dos años fue portada de todos los periódicos hace dos años. ¿Diríamos que el acaecimiento que más ha impresionado a Juanito ofrece alguna explicación del acaecimiento portada de todos los periódicos hace dos años? Desde luego diríamos que no la proporciona, a menos que sepamos que con el primer acaecimiento nos referimos al huracán Mitch y con el segundo a la muerte de miles de personas en Centroamérica hace dos años. Pero aún hay más, y esto es lo realmente importante para lo que nos ocupa, tampoco diríamos que existe ninguna relación causal entre esos acaecimientos si desconociéramos esas últimas descripciones de los mismos a través de las cuales se muestran subsumidos bajo la ley causal pertinente.

La lección importante que hay que extraer de estos ejemplos es la siguiente. No consideraremos que dos acaecimientos están vinculados por una relación de causa-efecto (no tendremos ninguna razón para ello) a menos que se nos presenten mediante las descripciones que los muestran subsumidos bajo lo que estimamos una ley causal. Llamémoslo el principio P.

Volvamos nuevamente al ejemplo anterior sobre Santiago Salvador. Es crucial observar que, de acuerdo con la tesis del anomalismo mental, la relación entre las razones de Salvador descritas como tales, es decir, en términos mentales, y su acción 
de arrojar las dos bombas es como la relación entre el acaecimiento que más ha impresionado a Juanito, descrito como tal, y el acaecimiento portada de todos los periódicos hace dos años, descrito como tal. Es decir, las razones de Salvador y su acción se presentan mediante descripciones que no muestran a esos acaecimientos subsumidos bajo ninguna ley causal, según se desprende del monismo anómalo. Por esa razón, aplicando el principio P, y razonando del mismo modo que en el caso análogo del huracán, deberíamos considerar que no hay vínculo causal entre ellos, o al menos deberíamos admitir que no tenemos ninguna buena razón para pensar que lo hay, como no la tenemos para pensar que la hay entre el acaecimiento que más ha impresionado a Juanito y el acaecimiento portada de todos los periódicos hace dos años, en ausencia de la información pertinente. ${ }^{23}$ Ahora bien, como sí parecemos pensar que hay un vínculo causal, en ciertos casos, entre razones para actuar y acciones — es más, como que el hecho de que juzguemos ciertas racionalizaciones como explicaciones correctas de acciones depende de que nos parezca que lo hay_-, lo que se desprende de la doctrina del anomalismo mental es que tal impresión es siempre errónea, pues proviene sin duda, en virtud del principio $\mathrm{P}$, de nuestra creencia falsa de que existen leyes causales psicofísicas o psicológicas. Pero en ese caso desaparece la razón para creer en la premisa 2.1, es decir, en la interacción causal entre acaecimientos mentales y acaecimientos físicos y con ella la justificación en favor del monismo físico. Así pues, hemos mostrado lo que perseguíamos: no hay modo de compatibilizar los argumentos de Davidson en favor del monismo físico con la tesis del anomalismo mental. No hay modo, pues, de hacer inteligible la defensa davidsoniana del monismo anómalo.

Recientemente, Davidson se ha defendido de las críticas que ha ido recibiendo el monismo anómalo a lo largo de los últimos años afirmando que tal posición sólo es incompatible con la existencia de leyes psicofísicas (o psicológicas) estrictas, pero no con

$23 \mathrm{Ni}$ que decir tiene que en el caso de las racionalizaciones desconocemos las descripciones físicas de las razones para actuar que supuestamente mostrarían tales razones subsumidas junto con la acción correspondiente bajo una ley causal física. 
la existencia de leyes psicofísicas (o psicológicas) no estrictas. ${ }^{24}$ ¿No podría ser éste un modo de resolver el problema mencionado en el párrafo anterior? Aparentemente sí, pues la existencia de leyes psicofísicas no estrictas podría en principio garantizar que existieran explicaciones causales correctas de acciones a partir de acaecimientos mentales. Quisiera terminar esta sección examinando brevemente esta posibilidad.

En primer lugar, para que esta posibilidad pueda tener éxito para superar el problema que hemos planteado, debería modificarse el análisis ofrecido antes acerca de lo que es una explicación causal, de modo que puedan considerarse explicaciones causales de un acaecimiento $a$ descrito mediante una descripción $d$ las explicaciones en las cuales los acaecimientos del explanans muestren a través de sus descripciones que están subsumidos con $a$ descrito mediante $d$ bajo una ley causal no necesariamente estricta.

El problema que presenta esta nueva elucidación de la noción de explicación causal es que sólo parece poder justificarse mediante una modificación análoga del Principio del Carácter Nomológico de la Causalidad, PCNC, en favor de otro, PCNC', que diga que siempre que un acaecimiento $a$ está vinculado causalmente con otro acaecimiento $b$, entonces existen descripciones de $a$ y $b$ mediante las cuales ambos se muestran subsumidos bajo una ley causal no necesariamente estricta. Ahora bien, si abandonamos el principio PCNC en favor del principio PCNC' entonces el argumento davidsoniano en favor del monismo físico, el argumento 2, deja de funcionar. La razón para ello es que ese argumento, como hemos visto, hacía un uso crucial del principio PCNC. En efecto, recordemos que se argumentaba que al haber una relación causal entre ciertos acaecimientos mentales y acaecimientos físicos, el PCNC exigía que hubiera descripciones de los mismos que los mostrasen subsumidos bajo una ley causal estricta. Finalmente, al no haber leyes psicofísicas estrictas se concluía que tenía que haber descripciones físicas no espurias de esos acaecimientos mentales y, por tanto, que en definitiva

24 Véase Davidson 1992. 
eran físicos. Ahora bien, puesto que PCNC' sólo exige que existan descripciones que muestren a los acaecimientos vinculados causalmente subsumidos bajo una ley causal no necesariamente estricta y puesto que existen leyes psicofísicas no estrictas, no podemos en este caso concluir que existen descripciones físicas no espurias de los acaecimientos mentales y, por consiguiente, que son acaecimientos físicos.

El segundo problema que presenta la nueva posición de Davidson como una posible solución al problema que hemos planteado es el siguiente. Hemos visto que según Davidson existen, de modo excepcional, razones a priori para descartar la posibilidad de que haya leyes psicofísicas o psicológicas estrictas. Como se recordará, tales razones tienen que ver con un principio constitutivo de los conceptos mentales, el requisito de racionalidad, que no es constitutivo de los conceptos físicos. Uno se pregunta entonces por qué estas mismas razones a priori no permiten descartar en cambio la posibilidad de leyes psicofísicas o psicológicas no estrictas. Dicho de otro modo, la dificultad es que si esas razones a priori no son obstáculo para la existencia de leyes psicofísicas o psicológicas no estrictas no se entiende que puedan serlo para la existencia de leyes psicofísicas o psicológicas estrictas. Una ley no estricta se distingue de una estricta solamente en que se le permiten excepciones, pero sustenta contrafácticos y se ve confirmada por sus instancias positivas igual que una ley estricta. Si el requisito de racionalidad no impide la existencia de este tipo de leyes, no debería impedir tampoco la existencia de leyes estrictas. En nuestra opinión estas razones bastan para descartar la defensa reciente de Davidson del monismo anómalo como una posible respuesta al problema que nosotros le hemos planteado. $^{25}$

25 Dicho sea de paso, no deja de mover a la reflexión el hecho de que absolutamente todo el mundo intepretara el monismo anómalo como una doctrina contraria a la existencia de leyes psicológicas o psicofísicas estrictas o no, cometiendo un error de interpretación, según Davidson. Véanse, como prueba de ello, la cantidad de artículos en las recopilaciones LePore y McLaughlin 1985 y Vermazen y Hintikka 1985. 


\section{Conclusiones}

Hasta ahora puede parecer que nuestro trabajo tiene un tono marcadamente crítico. Ha consistido en señalar una inconsistencia en el razonamiento davidsoniano en favor del monismo anómalo, en particular, entre el argumento en favor de la tesis $\mathrm{T}$ y la tesis del anomalismo de lo mental. Sin embargo, a mi juicio hay lecciones filosóficas interesantes que extraer de la crítica a Davidson que hemos planteado con interesantes implicaciones para la filosofía de la mente y de la acción. Así pues, querría cerrar este trabajo destacando el aspecto positivo de nuestra crítica.

A nuestro juicio, la principal lección que podemos extraer de nuestra discusión es que el argumento que Davidson ofreció en "Actions, Reasons and Causes" en favor de lo que hemos llamado la tesis $\mathrm{T}$ - esto es, la tesis de que la noción de causa que se utiliza en la comprensión de fenómenos naturales es también central en nuestra comprensión de las acciones intencionadas-, es mucho más potente de lo que el propio Davidson supuso. Muestra mucho más de lo que imaginó, y de hecho algo que no parece compatible con la tesis misma del monismo anómalo. Lo que muestra ese argumento es, esencialmente, que nuestro concepto de racionalización es el concepto de una explicación causal, en cuanto tal, del mismo género que las explicaciones causales que se utilizan en la explicación causal de fenómenos naturales en los que no interviene la voluntad humana. Las dos importantes diferencias estriban en que se trata de un tipo de explicaciones causales en las que se apela a causas mentales y en que las causas mentales deben guardar con la acción que explican ciertas relaciones de coherencia que la hagan inteligible o comprensible a nuestros ojos.

Pero estas diferencias, con ser importantes, no deben hacernos perder de vista lo que hay de común entre esas explicaciones. Lo que hay de común es que las concebimos en todo caso como explicaciones causales y que ello es así lo muestra a las claras el argumento de Davidson al hacernos ver que consideramos el vínculo causal entre los acaecimientos mentales atribuidos

- supuestas causas - y la acciones — supuestos efectos de los 
primeros - como una condición de corrección de dichas explicaciones. Exactamente lo mismo que hacemos con explicaciones causales de fenómenos naturales que no tengan que ver con la acción humana intencionada.

En virtud del principio del carácter nomológico de la causalidad, PCNC, que concibamos las racionalizaciones como explicaciones causales y que en ocasiones las juzguemos correctas significa que asumimos que existen leyes psicofísicas o psicológicas que vinculan acaecimientos mentales, descritos como tales, con acciones intencionadas. Nosotros, a diferencia de un davidsoniano consecuente, sí somos partidarios de la versión debilitada de este principio, que hemos llamado $\mathrm{PCNC}^{\prime}$. Es decir, la tesis de que las racionalizaciones son explicaciones causales, que algunas de ellas son correctas y que en una explicación causal se muestra el vínculo nómico entre explanans y explanandum no nos compromete con la existencia de leyes psicofísicas o psicológicas estrictas, sino sólo con la existencia de leyes psicofísicas o psicológicas, sean estrictas o no.

Por supuesto, hay dos modos de evitar las conclusiones del párrafo anterior. Un modo es declarar falso el principio del carácter nomológico de la causalidad, incluso en su versión debilitada que proponemos; el otro modo es declarar que, contra nuestro mejor juicio, todas las racionalizaciones de acciones intencionadas sin excepción constituyen explicaciones causales erróneas, al no haber leyes psicofísicas de ningún tipo.

Por esta razón es por lo que consideramos, como ya avanzamos al principio de este trabajo, que el argumento de Davidson, mucho más potente de lo que él mismo intuyó, ayuda en mucho a clarificar el panorama actual de la filosofía de la mente, pues nos deja sólo dos alternativas a la tesis de que existen leyes psicológicas o psicofísicas (estrictas o no). La primera es, como se dijo, negar el principio del carácter nomológico de la causalidad en cualquier versión; y la segunda, negar que pueda haber racionalizaciones correctas (es decir, que constituyan explicaciones correctas de alguna acción).

Querríamos concluir diciendo algo acerca de ambas alternativas. Por lo que respecta a la primera, antes de descartar el principio PCNC o $\mathrm{PCNC}^{\prime}$, debería tenerse en cuenta el doble 
papel teórico que este principio desempeña en metafísica. Ese doble papel es el siguiente. Por un lado, nos permite una elucidación de la noción, clave en metafísica, de explicación causal o, de modo más general, de explicación nomológica, con fuerza modal. La idea de que la corrección de esas explicaciones depende de leyes explica perfectamente su alcance modal, y las inferencias que los científicos suelen hacer a partir de ellas. El segundo papel tiene que ver con los tipos de usos del término 'causa' que Davidson aisló perfectamente. Por recurrir al ejemplo anterior en el enunciado 'el huracán Mitch causó la muerte de miles de personas en Centroamérica' se habla de causas en un sentido distinto que en el enunciado 'el acaecimiento que más ha impresionado a Juanito causó el acaecimiento portada de todos los periódicos hace dos años'. El PCNC, o en su defecto $\mathrm{PCNC}^{\prime}$, explican perfectamente en qué consiste esta diferencia, y nosotros al menos desconocemos un modo alternativo de explicarla.

Por lo que respecta a la segunda alternativa, resulta ocioso señalar un punto sobre el que se ha insistido constantemente en las últimas décadas: declarar toda racionalización como una explicación errónea hace totalmente misteriosa la posibilidad humana de comunicación con otros, de coordinación con otros, etc., así como infundada cualquier norma ética o jurídica.

Parece claro que cualquiera de estas dos alternativas a la tesis de que existen leyes psicofísicas o psicológicas resulta poco prometedora. En cualquier caso, nuestra investigación parece conducirnos, y ése es a nuestro juicio su mayor mérito, a la conclusión de que una de ellas debe ser aceptada, de que no hay más alternativas. A este respecto, resulta instructivo notar que el monismo anómalo es incompatible con las tres, y por esa razón deviene una posición imposible de sostener.

\section{BIBLIOGRAFÍA}

Davidson, Donald, 1993, “Thinking Causes", en Heil y Mele (comps.), Mental Causation, Oxford University Press, Oxford, pp. 3-17.

_, 1980 , Essays on Actions and Events, Oxford University Press, Oxford. [Versión en castellano: Ensayos sobre acciones y sucesos, 
trads. Olbeth Hansberg, José Antonio Robles y Margarita Valdés, Instituto de Investigaciones Filosóficas-UnAM, México, 1995.]

_ _ 1974, "Psychology as Philosophy", reeditado en Davidson 1980, pp. 229-239.

—_, 1970, "Mental Events", reeditado en Davidson 1980, pp. 207223.

—_, 1967, "Causal Relations", reeditado en Davidson 1980, pp. 149162.

, 1966, "Emeroses by Other Names", reeditado en Davidson 1980, pp. 225-227.

——, 1963, "Actions, Reasons, and Causes", reeditado en Davidson, Essays on Actions and Events, pp. 3-19. (Las referencias de páginas de este y los sucesivos artículos de Davidson corresponden a esta edición.)

Goodman, Nelson, 1955, "The New Riddle of Induction", Fact, Fiction and Forecast, Harvard University Press, Cambridge, Mass.

Jackson, Frank, 1986, "What Mary Didn't Know", The Journal of Philosophy, vol. 83, pp. 291-295.

Kim, Jaegwon, 1985, "Psychophysical Laws", en LePore y McLauglin (comps.), 1985.

Kripke, Saul A., 1981, Naming and Necessity, Harvard University Press, Cambridge, Mass. [Versión en castellano: El nombrar y la necesidad, trad. Margarita Valdés, Instituto de Investigaciones Filosóficas-Unam, México, 1995.]

LePore, E. y B. McLauglin (comps.), 1985, Actions and Events. Perspectives on the Philosophy of Donald Davidson, Basil Blackwell, Oxford.

Nagel, Thomas, 1974, "What Is It Like to Be a Bat", The Philosophical Review, vol. 83, pp. 435-450.

Vermazen, B. y M. Hintikka (comps.), 1985, Essays on Davidson. Actions and Events, Clarendon Press, Oxford.

Recibido: 7 de septiembre de 2000 Aceptado: 8 de febrero de 2001 